\title{
Criminologie
}

\section{La notion de risque dans la gestion pénale}

\section{Pierre Landreville et Germain Trottier}

Volume 34, numéro 1, printemps 2001

La notion de risque dans la gestion pénale

URI : https://id.erudit.org/iderudit/004754ar

DOI : https://doi.org/10.7202/004754ar

Aller au sommaire du numéro

Éditeur(s)

Les Presses de l'Université de Montréal

ISSN

0316-0041 (imprimé)

1492-1367 (numérique)

Découvrir la revue

Citer cet article

Landreville, P. \& Trottier, G. (2001). La notion de risque dans la gestion pénale. Criminologie, 34(1), 3-8. https://doi.org/10.7202/004754ar d'utilisation que vous pouvez consulter en ligne.

https://apropos.erudit.org/fr/usagers/politique-dutilisation/ 


\title{
La notion de risque dans la gestion pénale
}

\author{
Pierre Landreville \\ Professeur \\ École de criminologie \\ Université de Montréal \\ pierre.landreville@umontreal.ca \\ Germain Trottier \\ Professeur \\ École de service social \\ Université Laval \\ germain.trottier@svs.ulaval.ca
}

Pour résoudre une conjoncture problématique, les décideurs ont l'habitude de recourir au savoir spécialisé des experts pour fonder leurs opinions. Théoriquement parlant, l'expert a pour fonction d'être un auxiliaire averti, au service du décideur mais en pratique, on constate que les avis des experts créent de plus en plus les normes et définissent en quelque sorte les conduites à adopter et éviter. En ce qui a trait à la délinquance juvénile, les recherches démontrent que les recommandations des experts sont appliquées par les magistrats dans $95 \%$ des cas requis. L'expertise est devenue comme une nouvelle religion des temps modernes (Castel, 1981) et la gestion du risque constitue un de ces champs d'expertise majeure depuis quelques décennies (Malenfant, 1998).

Le risque occupe une position centrale dans le champ social contemporain et fait l'objet de vives discussions dans les communautés scientifiques. Y a-t-il plus de risques aujourd'hui qu' autrefois ? Risque pour qui et risque de quoi ? Comment appréhender le risque, le mesurer, le 
prédire ? Pourquoi étudier les facteurs de risque, et agir sur les situations à risque pour en prévenir les effets nuisibles ? Voilà autant de questions qu'il y a lieu de se poser dans cette « société du risque » (Beck, 1992).

Notre rapport au risque et la notion de risque ont profondément changé au cours du siècle dernier. Longtemps associés à la catastrophe, à la fatalité et souvent attribués au destin ou à des forces externes, d'origine divines ou autres, les risques ne sont plus totalement imprévisibles. Les développements scientifiques ont transformé notre vision du monde et nous essayons de plus en plus de comprendre les phénomènes et de les maîtriser, d'où la tendance à vouloir anticiper les risques inhérents aux événements et en atténuer les conséquences néfastes. L'amélioration de la durée et de la qualité de la vie dans nos sociétés, la couverture offerte par les assurances privées ou publiques contre les conséquences de la maladie, de la pauvreté extrême, du chômage, des accidents de la route, etc. ont aussi transformé la conception que nous avons de la vie qui devient de plus en plus précieuse et de plus en plus perçue comme un «droit ». Cette nouvelle sensibilité crée cependant de nouvelles attentes. Les dangers contre la vie deviennent de plus en plus intolérables, ils semblent être de plus en plus nombreux, de plus en plus menaçants et la demande de protection sociale se fait plus pressante.

Comme le mentionne J. Pratt dans l'article de ce numéro de la revue, ces attentes se retrouvent aussi dans le domaine pénal où l'on exige que l'État nous protège des délinquants dits dangereux, des " prédateurs sexuels », des délinquants violents. Ici aussi, les avis des experts sont requis pour évaluer, prévenir les risques, pour déterminer et gérer les situations à risque et les " groupes à risques".

C'est dans ce contexte que la revue Criminologie a jugé bon de consacrer ce numéro à la notion de risque dans le système pénal. Nous abordons successivement les techniques de l'évaluation et de la prédiction du risque, leur impact sur les pratiques et les politiques correctionnelles, et enfin la signification sociale de ces transformations.

Tout d'abord J. Proulx et P. Lussier, deux spécialistes de l'évaluation et du traitement des délinquants sexuels, présentent l'état des connaissances en ce qui concerne la prédiction de la récidive chez ce type d'agresseurs. G. Côté, un clinicien et un chercheur spécialisé dans le domaine des troubles mentaux sévères, poursuit quant à lui une réflexion très stimulante sur les instruments actuariels et l'apport de la démarche clinique qui prend en compte des aspects dynamiques dans l'évaluation des risques de comportements violents. 
Par la suite, deux sociologues K. Hannah-Moffat et M. Shaw font une analyse critique de l'utilisation des instruments d'évaluation du risque dans le système correctionnel fédéral du Canada. Ces auteures soulèvent les problèmes méthodologiques, éthiques et politiques engendrés par l'utilisation d'instruments, construits et validés avec des populations d'hommes «blancs ", pour évaluer des femmes et des membres des communautés ethno-culturelles, en particulier des autochtones. D. Robert, quant à elle, s'intéresse à la transformation de la loi sur la mise en liberté sous condition au Canada. Elle montre comment la tendance duale (bifurcation, selon l'expression de Bottoms, 1977) opère de plus en plus sur la base du critère de risque.

Enfin, J. Pratt, un criminologue travaillant en Nouvelle-Zélande, examine les transformations des législations sur les délinquants dangereux, principalement dans les pays de Common-law, montre comment la notion de dangerosité est un construit social et comment ces modifications participent à la transformation du contrôle social dans les sociétés modernes.

La notion de dangerosité et la prédiction de la récidive ou des comportements violents ne sont pas nouvelles dans le système pénal. Il $\mathrm{y}$ a, depuis le début du $\mathrm{XX}^{\mathrm{e}}$ siècle, sous une forme ou sous une autre, des lois spéciales concernant les «délinquants dangereux » (Pratt dans ce numéro). Les experts et les décideurs pénaux tentent de prédire les comportements futurs des délinquants soit pour les détenir pendant les procédures pénales, soit pour décider de la peine à imposer ou du moment de la remise en liberté. Si ces prédictions sont ordinairement intuitives, on a parfois recours à l'expert clinicien et de plus en plus aux instruments actuariels. Récemment, ces outils ont pris de plus en plus d'importance grâce à une certaine remise en question de la capacité de prédiction des cliniciens et aux ordinateurs qui ont permis l'émergence d'une nouvelle génération de techniques statistiques plus complexes et sophistiquées.

Le recours accru aux techniques de prédiction, à la notion de risque dans l'évaluation et la gestion de populations délinquantes soulèvent cependant des questions méthodologiques, épistémologiques, légales et politiques qui sont abordées dans ce numéro. Du point de vue méthodologique, J. Proulx et $\mathrm{P}$. Lussier mentionnent les problèmes liés au choix de prédicteurs et de critères de la récidive fiables et valides auxquels sont confrontés tant les cliniciens que ceux qui élaborent des instruments actuariels, ainsi que les inconsistances dans la durée des 
périodes d'épreuve. Dans le cas des délinquants sexuels ou des délinquants violents, les taux de récidive sont relativement faibles, ce qui rend encore plus difficile l'exercice de prédiction, de sorte qu'il paraît souhaitable que la période d'épreuve soit assez longue pour augmenter le taux de base. Malheureusement, il est souvent demandé à l'expert d'évaluer les risques à court terme ce qui augmente la difficulté de l'exercice et rend problématique l'utilisation d'instruments actuariels pour évaluer la récidive sur de plus longues périodes.

G. Côté soulève, pour sa part, la question de la généralisation, particulièrement l'usage très discutable d'instruments de prédiction dans des populations différentes de celles qui ont servi à développer les instruments. Les objections de K. Hannah-Moffat et M. Shaw concernant l'utilisation d'instruments élaborés à partir d'un échantillon d'hommes «blancs » dans des populations féminines ou d'autochtones sont particulièrement pertinentes à cet égard.

Toutes ces remarques méthodologiques entraînent nécessairement des questionnements éthiques sur l'usage de ces instruments. G. Côté ajoute qu'il est problématique, de ce point de vue, de n'utiliser que des variables statiques, que des aspects qui ne se modifient pas à travers le temps alors que les personnes changent et que les situations dans lesquelles elles sont appelées à vivre se modifient. De plus, les instruments actuariels établissent les probabilités de récidive d'un groupe d'individus et ne peuvent tenir compte de la situation particulière de la personne en cause dans la prise de décision. Cet élément est d'autant plus important lorsqu'il s'agit de la prédiction d'un comportement rare qui produit inévitablement un nombre important de faux positifs, soit le fait d'identifier faussement des personnes comme de futures récidivistes ou des personnes à risque. Ce problème fondamental du point de vue éthique est généralement minimisé ou est effleuré succinctement pour la forme. Il faut aussi souligner les critiques d'Hannah-Moffat et M. Shaw selon lesquelles on doit remettre en question l'utilisation de facteurs structuraux sur lesquels les personnes n'ont aucun contrôle ou de variables qui peuvent être fortement teintées de jugements de classe, ethnocentriques ou même racistes.

Du point de vue éthique et légal on peut aussi ajouter que les évaluations actuarielles du risque sont le plus souvent acceptées par les décideurs, comme les commissions des libérations conditionnelles ou les juges, sans que les personnes concernées puissent réellement les remettre en question. Règle générale, ni eux, ni leurs représentants n’ont les 
compétences requises pour questionner la fiabilité et la validité des informations prises en considération ou la construction de l'instrument lui-même. Parfois l'instrument est élaboré de façon telle qu'il est difficile et même impossible de connaitre ces informations. De plus, l'expert qui l'a construit n'est pas là pour l'expliquer et le clinicien ou l'intervenant qui l'a utilisé n'est généralement pas en mesure de fournir les explications souhaitables (voir Price, 1997, à ce sujet). Enfin, la prépondérance accordée au comportement futur dans les décisions va à l'encontre d'un des principes fondamentaux de la détermination de la peine, le principe de proportionnalité.

D'un point de vue plus général, l'accent que l'on met sur l'évaluation des risques, sur la prédiction des comportements, entraîne (ou participe à) de profonds changements dans les priorités et les modalités de gestion pénale et à une nouvelle «technologie du pouvoir » (Pratt). Comme le mentionne D. Robert, on cherche de moins en moins à transformer les individus mais plutôt à gérer les risques qu'ils représentent. « Il s'agit de minimiser les pertes, de diminuer les opportunités d'infractions et non de transformer les infracteurs ou la société en profondeur », "l'unité de gestion passe de l'individu aux groupes. On cherche à les catégoriser, les classifier, les hiérarchiser. Des trajectoires institutionnelles sont tracées pour chaque groupe conformément au danger qu'il représente. Le diagnostic et le traitement ont fait place respectivement à l'attribution d'un profil et à l'assignation administrative. » Pratt ajoute que cette information actuarielle concernant l'identification des risques a aussi un impact sur les victimes potentielles, auxquelles on demandera de modifier leurs comportements pour s'informer des risques, se protéger, faire des « choix rationnels » et diminuer leur risque de victimisation. Elles aussi devraient se « responsabiliser ».

Nous souhaitons que ces articles alimentent la réflexion et les débats sur la notion de risque dans le champ pénal, et qu'ils aideront tant les chercheurs que les intervenants à prendre une distance critique par rapport à cette nouvelle façon d'aborder les problèmes. Nous souhaitons également que ces articles permettent de saisir que les débats sur la prédiction et l'évaluation des risques ne portent pas uniquement sur l'efficacité mais aussi sur la signification sociale des transformations en cours. 


\section{Références}

BECK, U. 1992. Risk Society.Towards a New Modernity, Newbury Park : Sage Publications.

CASTEL, R. 1981. La gestion des risques. De l'anti-psychiatrie à l'après psychanalyse, Paris : Les Éditions de Minuit.

CulPITT, I. 1999. Social Policy and Risk, London : Sage Publications.

Feeley, M. et Simon, J. 1992. "The New Penology: Notes on the Emerging Strategy of Corrections and its Implications » Criminology 30 (4) : 449-474.

FeELEY, M. et Simon, J. 1994. « Actuarial Justice: the Emerging New Criminal Law », Pp. 173-201 in The Futures of Criminology, sous la direction de D. Nelken. London : Sage Publications.

Le Breton, D. 1996. Passions du risque, Paris : A. M. Métaillé.

MALENFANT, R. 1998. « Risque et gestion du social » Recherches sociographiques 39 (1).

PRICE, R. 1997. "On the Risks of Risk Prediction » The Journal of Forensic Psychiatry 3 (1) : 1-4. 